\title{
More than $45 \%$ of A549 and H446 cells are cancer initiating cells: Evidence from cloning and tumorigenic analyses
}

\author{
XIANGJIAO MENG, XIUWEN WANG and YAWEI WANG \\ Department of Chemotherapy, Oncology Center, Qilu Hospital, Shandong University, \\ No. 107 Wenhua Xi Road, Jinan 250012, P.R. China
}

Received October 20, 2008; Accepted December 17, 2008

DOI: $10.3892 /$ or_00000314

\begin{abstract}
Cancer stem cells have been isolated from human lung cancer and cancer cell lines. This study was designed to investigate the fraction of cancer stem cells in the A549 and H446 cell lines by cloning and tumorigenic analyses. We found that $>45 \%$ of A549 and H446 cells formed large clones that are able to generate subclones and subsequently give rise to xenograft tumors in the BALB/C-nude mouse. Both $\mathrm{CD}_{133^{+}}$and CD133- cells of A549 and H446 possessed stem cell characteristics and exposure to Hoechst 33342 affected the clonogenicity and proliferation of single A549 and $\mathrm{H} 446$ cell. Moreover, based on cell cycle phase distribution and growth curve, most A549 and H446 cells may not be stem cells, but they acquired stem cell characteristics when they were alone. Therefore, these cells that formed large clones should be called cancer initiating cells, not stem cells. Thus, we concluded that $>45 \%$ of A549 and H446 cells were cancer initiating cells, although many of them were neither $\mathrm{CD}_{133}{ }^{+}$cells nor side population cells.
\end{abstract}

\section{Introduction}

It was demonstrated that malignant tumors and cell lines of brain (1-3), breast (4), prostate (5), liver (6,7), pancreas (8), lung $(9,10)$ and colon $(11,12)$ contain cancer stem cells (CSCs). CSCs that regenerate and express stem cell markers are tumorigenic and resistant to chemotherapeutic drugs and irradiation (13-15). Two methods have been introduced to isolate cancer stem cells from lung cancer and cancer cell lines. In the first method, cancer stem cells were isolated through the stem cell marker CD133. Lung cancer CD133+ cells were able to grow indefinitely as tumor spheres in serum-free medium containing epidermal growth factor and basic fibroblast growth factor. The injection of $10^{4}$ lung cancer

Correspondence to: Dr Xiuwen Wang, Department of Chemotherapy, Oncology Center, Qilu Hospital, Shandong University, No. 107 Wenhua Xi Road, Jinan 250012, P.R. China

E-mail: wangxw12@yahoo.com

Key words: lung cancer cell line, clone and tumorigenic analyses, CD133, Hoechst 33342, cancer initiating cells
$\mathrm{CD}_{133^{+}}$cells into immunocompromised mice readily generated xenograft tumor phenotypically identical to the original tumor. Upon differentiation, lung cancer $\mathrm{CD} 133^{+}$cells acquired the specific lineage markers, while losing the tumorigenic potential with CD133 expression (9).

In the second method, side popoulation (SP) cells were isolated and characterized by flow cytometry and Hoechst 33342 dye efflux assays from six human lung cancer cell lines. Non-obese diabetic/severe combined immunodeficiency (NOD/SCID) mice xenograft experiments showed that SP cells were enriched in tumor-initiating capability compared with non-SP cells. Matrigel invasion assay showed that SP cells also have higher potential invasiveness. Further characterization of this SP phenotype revealed several stem cell properties. It was found that SP cells had the ability to regenerate a population resembling the original population. SP cells displayed elevated expression of ABCG2 as well as other ATP-binding cassette transporters and showed resistance to multiple chemotherapeutic drugs. Sixteen clinical lung cancer samples also displayed a smaller but persistent SP population (10).

However, recently it was reported that four of 15 cell lines derived from primary glioblastomas grew adherently in vitro and were driven by CD133- tumor cells that met stem cell criteria, and both $\mathrm{CD}_{133^{+}}$and $\mathrm{CD} 133^{-}$tumor cells were similarly tumorigenic in nude mice in vivo (16). It was also reported that both $\mathrm{CD}_{133^{+}}$and $\mathrm{CD} 133^{-}$metastatic tumor subpopulations formed colonospheres in cultures and were capable of long-term tumorigenesis in a NOD/SCID serial xenotransplantation model (17). Moreover, there are studies that Hoechst 33342 is cytotoxic and Hoechst 33342 staining itself may interfere with the clonogenicity of stem cells (18-20). This study was designed to investigate the fraction of cancer initiating cells (cells that are able to initiate and sustain tumor growth) in the A549 and H446 cell lines using cloning and population analyses without any classification based on CD133 or ABCG2.

\section{Materials and methods}

Cell culture. Human lung cancer cell lines A549 and H446 were obtained from American Type Culture Collection (ATCC) and maintained in culture medium recommended by ATCC. Cells were cultured in 1640 and $10 \%$ fetal bovine serum (FBS). Cells were incubated at $37^{\circ} \mathrm{C}$ under $95 \%$ air, 5\% $\mathrm{CO}_{2}$ and $100 \%$ humidity. 


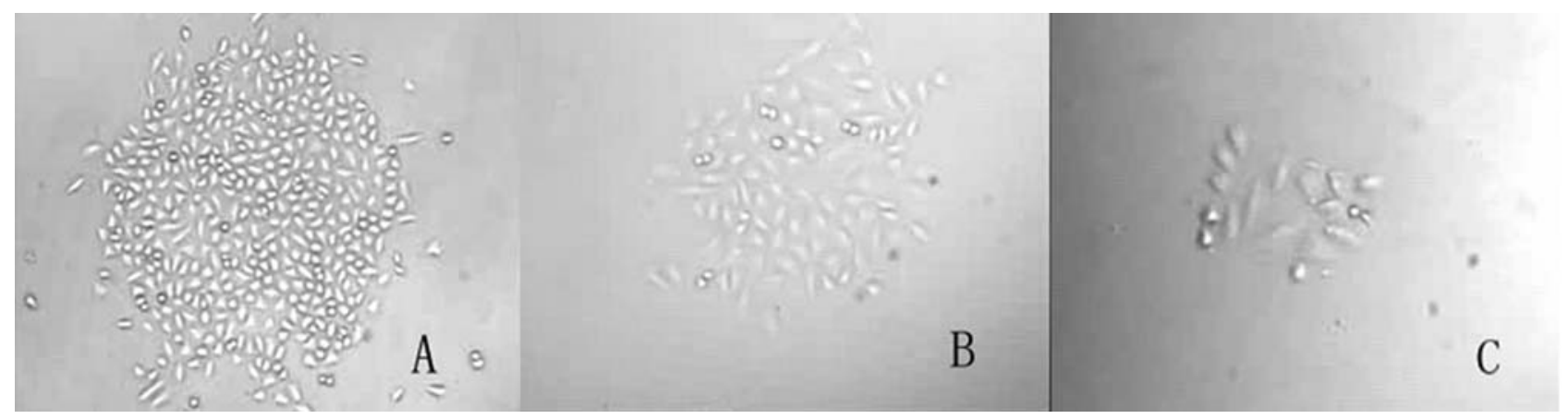

Figure 1. Clonogenic capacity of A549 single cells. (A) A large clone of A549 (x100). (B) A median clone of A549 (x100). (C) A small clone of A549 (x100).

Clonogenicity analyses. To determine the clonogenicity and regeneration ability of a cell, a clone formation assay was carried out as described with some modifications (18). Briefly, cells were harvested by $0.25 \%$ trypsin in logarithmic phase of growth and centrifuged at 1,000 rpm for $5 \mathrm{~min}$. The cells were resuspended in fresh medium, diluted to 10 cells $/ \mathrm{ml}$ using routine procedures of end-point dilution, seeded into 96-well plates (100 $\mu 1$ per well), and cultured in 1640 and $10 \%$ FBS. Wells containing no cell or more than one cell were excluded, and those with only one cell were marked and checked daily under a microscope. After one week, the clones were counted, dissociated and cultured similarly in new 96-well plates $(1$ cell/well) to generate subclones.

Tumorigenicity assay. Clones in the 96-well plates were digested with $0.25 \%$ trypsin and expanded in the same well. When the cells covered $70 \%$ of the well, they were transferred to 24-well plates and expanded. Then they were transferred to 6-well plates. Finally, the cells from one clone were cultured in $75 \mathrm{~cm}^{3}$ flasks. The clone cells were then injected into the left axillary fossa of 4-week-old BALB/C-nude mice (supplied by the Shanghai Experimental Animal Center, Chinese Academy of Sciences, Shanghai, China). The mice were examined visually. When the xenograft tumors grew to $\sim 8$ $\mathrm{mm}$ in diameter, the mice were sacrificed under deep anesthesia with pentobarbital. The tumors were then dissected, and one part was fixed with $4 \%$ paraformaldehyde. Paraffin sections were prepared following routine procedures for H\&E staining and immunohistochemical examination. The other part was subjected to mechanical and enzymatic dissociation. The digest was further sieved through a $100-\mu \mathrm{m}$ cell strainer to obtain single cell suspension for clone analyses.

Immunohistochemical assay. The expression of stem cell marker OCT-4 was examined by immunohistochemical assay. Xenograft tumor tissues were embedded in paraffin. Paraffin-embedded tissue sections were deparaffinized in xylene and were rehydrated through graded alcohol. Antigen retrieval was performed in sodium citrate buffer $(\mathrm{pH} \mathrm{6.0)}$ at $95-98^{\circ} \mathrm{C}$ for $10 \mathrm{~min}$. Hydrogen peroxide $(0.3 \%)$ was used to block the endogenous peroxidases for $10 \mathrm{~min}$. Tissue sections were incubated in goat serum as a blocking solution at $37^{\circ} \mathrm{C}$ for $30 \mathrm{~min}$. Sections were incubated with anti-OCT-4 (1:100 Cell Signaling Technology, Boston, USA) overnight at $4^{\circ} \mathrm{C}$. After the tissue sections were washed with PBS (phosphate-buffered solution), the secondary antibody was applied and incubated for $30 \mathrm{~min}$ at $37^{\circ} \mathrm{C}$. Finally, the slides were soaked in HRP substrate buffer for $10 \mathrm{~min}$, incubated with DAB solution until brown color appeared, washed with deionized water, and counterstained with hematoxylin.

The clonogenicity of $\mathrm{CD}_{133^{+}}$cells and CD133- cells. To determine whether CD133- cells (cells negative for CD133) of A549 and H446 possess the characteristics of CSCs, we isolated the cells by magnetic separation. Single cells were magnetically labeled with CD133 cell isolation kit (Miltenyi Biotec, Bergisch Gladbach, Germany) in the dark at $4^{\circ} \mathrm{C}$ for $30 \mathrm{~min}$ and separated by double passage through magnetic separation instruction of Miltenyi. CD133 expression of A549 and H446 were assessed using anti-CD133-2 phycoerythrin antibody (Miltenyi Biotec). After magnetic bead separation, separated cells were assessed by flow cytometry for purity. $\mathrm{CD}_{133^{+}}$cells (cells positive for CD133) and CD133- cells were subsequently tested for clonogenicity, following the methods described above.

Effect of Hoechst 33342 on cancer stem cells. To determine whether Hoechst 33342 staining could affect the clonogenicity of cells, A549 and H446 cells were incubated with Hoechst 33342 (Sigma, MO, USA) at a concentration of $5 \mu \mathrm{g} / \mathrm{ml}$ for $90 \mathrm{~min}$ at $37^{\circ} \mathrm{C}$, collected by centrifugation, and resuspended in Hoechst 33342-free medium. Trypan blue was used to determine the viability of the Hoechst 33342-free cells (control) and Hoechst 33342-treated cells. Clonogenic analyses of Hoechst 33342-free cells and Hoechst 33342-treated cells were performed as described above.

Cell cycle study. The distribution of cell cycle phase was assayed by flow cytometry. A549 and H446 cells (1x106) were harvested by treatment with $0.25 \%$ trypsin and centrifugation, washed with ice-cold PBS and resuspended in $3 \mathrm{ml}$ (70\%) ice-cold alcohol overnight. Then the cells were washed twice with ice-cold PBS and resuspended in $50 \mu \mathrm{g} / \mathrm{ml}$ propidium iodide (Sigma) and $10 \mu \mathrm{g} / \mathrm{ml}$ RNase A (Sigma) and incubated at $4^{\circ} \mathrm{C}$ in the dark for $30 \mathrm{~min}$. FACStar was used to measure the percentage of cell population in each phase of the cell cycle.

Cell proliferation assay. We used 3-[4,5-demethylthiazol2,5-diphenyl-2H-tetrazolium bromide] (MTT) assay to 


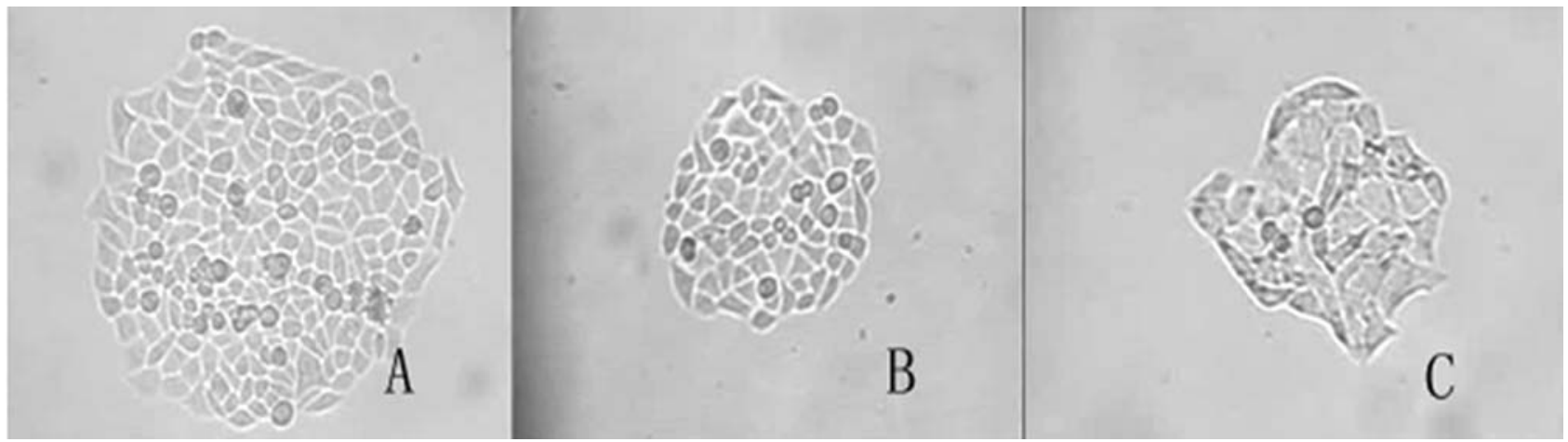

Figure 2. Clonogenic capacity of H446 single cells. (A) A large clone of H446 (x200). (B) A median clone of H446 (x200). (C) A small clone of H446 $(\mathrm{x} 200)$.

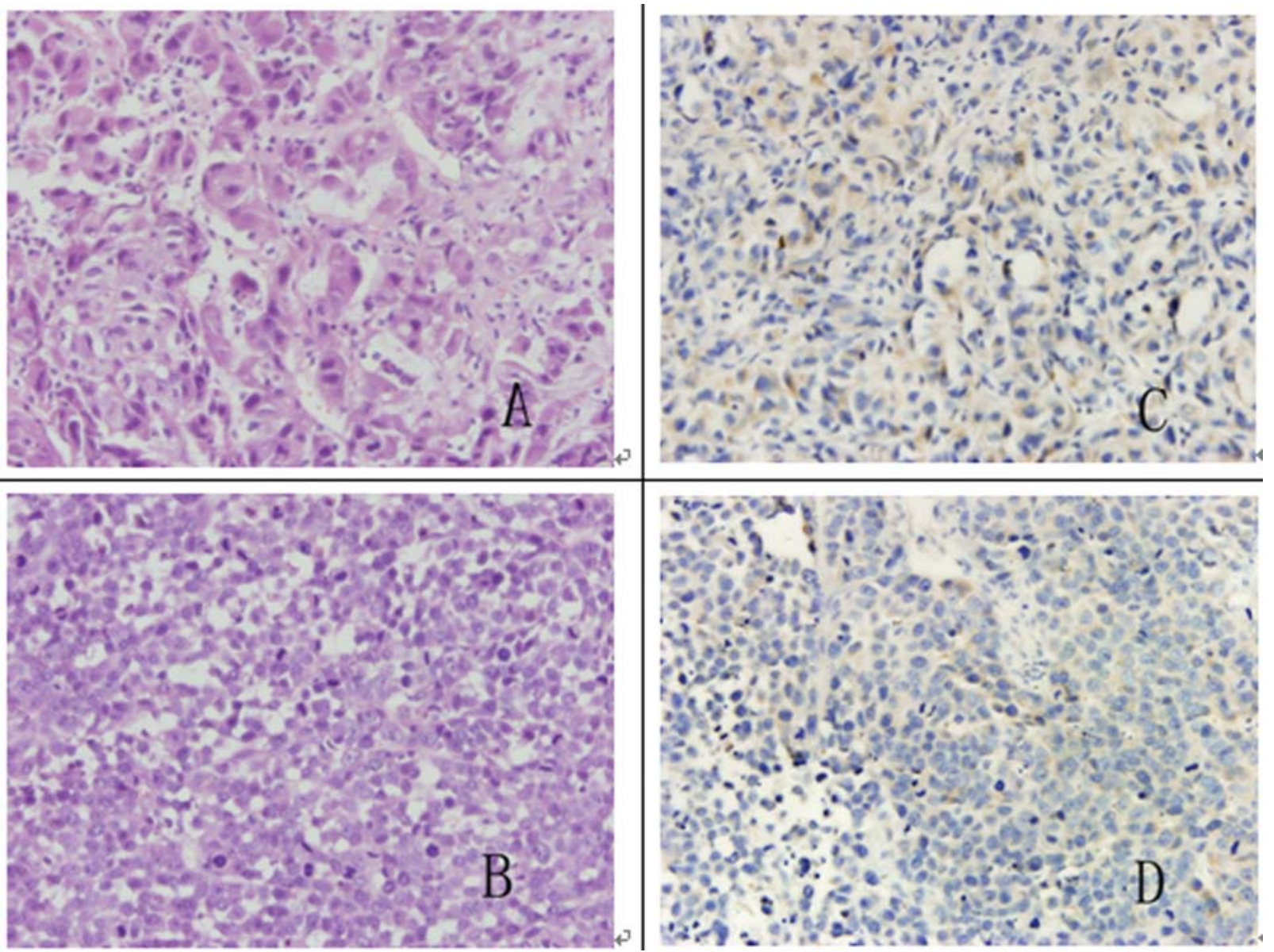

Figure 3. Tumorigenic capacity of A549 and H446 single cells. (A) H\&E, staining of A549 xenograft tumor. (B) H\&E, staining of H446 xenograft tumor. (C) OCT-4 expression of A549 xenograft tumor. (D) OCT-4 expression of H446 xenograft tumor.

determine the growth curves of different clones of A549 and H446 cell lines. Six clones were chosen by random. Clones in the 96-well plates were digested with $0.25 \%$ trypsin and expanded in the same well. Five days later, they were transferred to 24-well plates and expanded. Then they were transferred to 6 -well plates. When the cells covered $70 \%$ of the well, clone cells were dissociated, adjusted to $1.25 \times 10^{4}$ cells $/ \mathrm{ml}$, and seeded into seven 96-well plates with $200 \mu 1$ per well. A MTT assay was done daily (one plate each time) for 7 days. First, $20 \mu \mathrm{l}$ MTT $(5 \mathrm{mg} / \mathrm{ml})$ was added to each well, and the cells were incubated at $37^{\circ} \mathrm{C}$ for $4 \mathrm{~h}$. Then, the culture medium was removed and $150 \mu 1$ DMSO was added to each well. After shaking for $10 \mathrm{~min}$, the absorbance at $550 \mathrm{~nm}$ of each well was measured on a plate reader. Growth curves were obtained by plotting the absorbance vs. time.

Statistical analyses. Data are expressed as the mean \pm SD. Student's t-tests were used to compare the difference between two groups. $\mathrm{P}<0.05$ was accepted as statistically significant. 

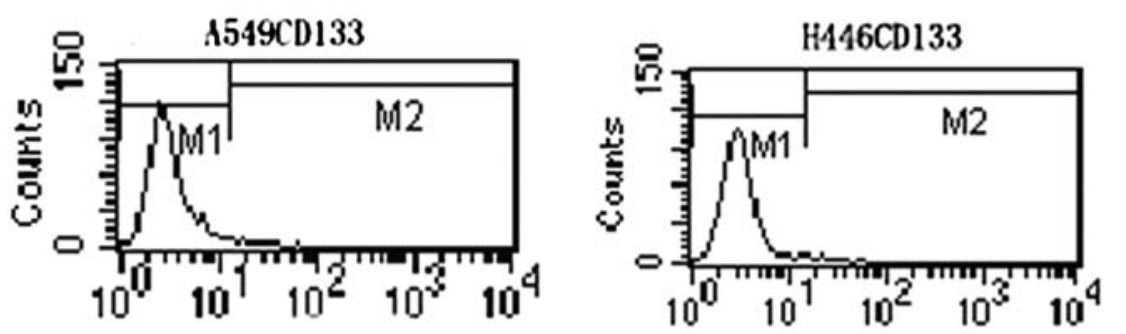

A
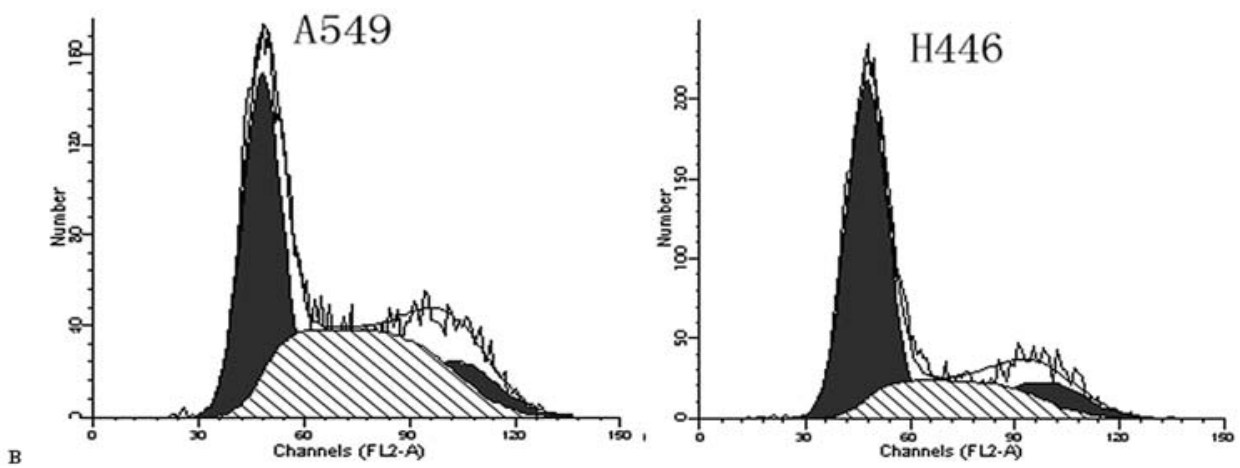

Figure 4. Flow cytometry study. (A) The CD133 fraction of A549 (0.98\%) and the CD133 fraction of H446 (1.00\%). (B) The fractions of G1, G2 and S phase of $\mathrm{A} 549(43.39,13.65$ and $42.96 \%)$ and the fraction of G1, G2 and S phase of H446 $(62.75,13.29$ and $23.96 \%)$

\section{Results}

Clone formation of A549 and H446 cells. Both A549 and H446 single cells can form three kinds of clones in the serum-containing medium in one week, i.e. large clones ( $\mathrm{L}>100$ cells), median clones (M, 50-100 cells) and small clones $(\mathrm{S}<50$ cells). The cells in large clones were homogeneous in cell morphology and highly proliferate. The cells in the small clones seemed fragmented, even the viable cells were less healthy than the cells in large clones (Figs. 1 and 2). The formation rates of large, median and small clones of A549 were $46.3 \pm 3.6,25.9 \pm 4.9$ and $27.8 \pm 4.9 \%$, respectively. The formation rates of large, median and small clones of H446 were $47.5 \pm 8.9,27 \pm 9,25.13 \pm 8 \%$, respectively.

After being dissociated and cultured similarly in new 96-well plates $(1 \mathrm{cell} / \mathrm{well})$, the cells from the small clones cannot form new clones and they underwent apoptosis instead in the second generation. The cells from the median clones formed a few median clones in the first generation and formed mostly small ones in the second generation; they underwent apoptosis and formed a few small clones in the third generation; all the cells underwent apoptosis in the fourth generation. The cells from the large clones formed the three kinds of clones even in the tenth generation, and the percentage of large clones was a little higher in the first generation but remained the same in the other generations. These results showed that the cells that can form large clones, which account for $46.3 \%$ of A549 cells and $47.5 \%$ of H446 cells, have the regeneration ability.

Cells that can form large clones are tumorigenic. The large clones and median clones were expanded for injection into nude mice to test for tumorigenicity. However, 20 days later, only the cell forming the large clone was expanded to $10^{6}$ cells, which were then injected into nude mouse. There was not enough number of cells growing from median clones for injection. After 10 days, tumor was observed at each of the injection sites. Typical morphological characteristics of lung cancer were evident in the tumor tissue sections (Fig. 3A and $\mathrm{B}$ ). The cells from xenograft tumor were able to form three kinds of clones as cells before injected.

Expression of the stem cell marker OCT-4 in xenograft tumors. It was reported that OCT-4 acts as a multifunctional factor in cancer and stem cell biology (21) and OCT-4 increases the malignancy of ES cells in a dose dependent manner (22). Our immunohistological examination showed that $40 \%$ of A549 cells expressed OCT-4, but $<10 \%$ of $\mathrm{H} 446$ cells expressed OCT-4. The intensity of OCT-4 immunostaining of A549 was much stronger than that of H446 (Fig. 3C and D).

The clone formation of $\mathrm{CDI}_{3} 3^{+}$and CD133- cells. The fractions of CD133 expressing cells of A549 and H446 were 0.98 and $1.00 \%$, respectively (Fig. 4A). The $\mathrm{CD} 133^{+}$cells and CD133- cells were separated and cultured separately. Both CD133+ cells and CD133- cells of A549 and H446 formed the three kinds of clones and the formation rates were just a little lower than that of unseparated cells, likely due to the effects of the separation process (Fig. 5).

Hoechst 33342 inhibited the clonogenicity of A549 and H446 cells. We showed that the viability of the cells were significantly influenced by Hoechst 33342 staining. A549 and H446 cells were incubated with Hoechst 33342 for 90 min and cultured in 96 -well plates. Seven days later, only $78 \%$ of Hoechst 33342-treated A549 cells formed clones, but $99 \%$ of Hoechst 33342-free A549 cells formed clones. Similarly, only 83\% of Hoechst 33342-treated H446 cells formed clones, but 

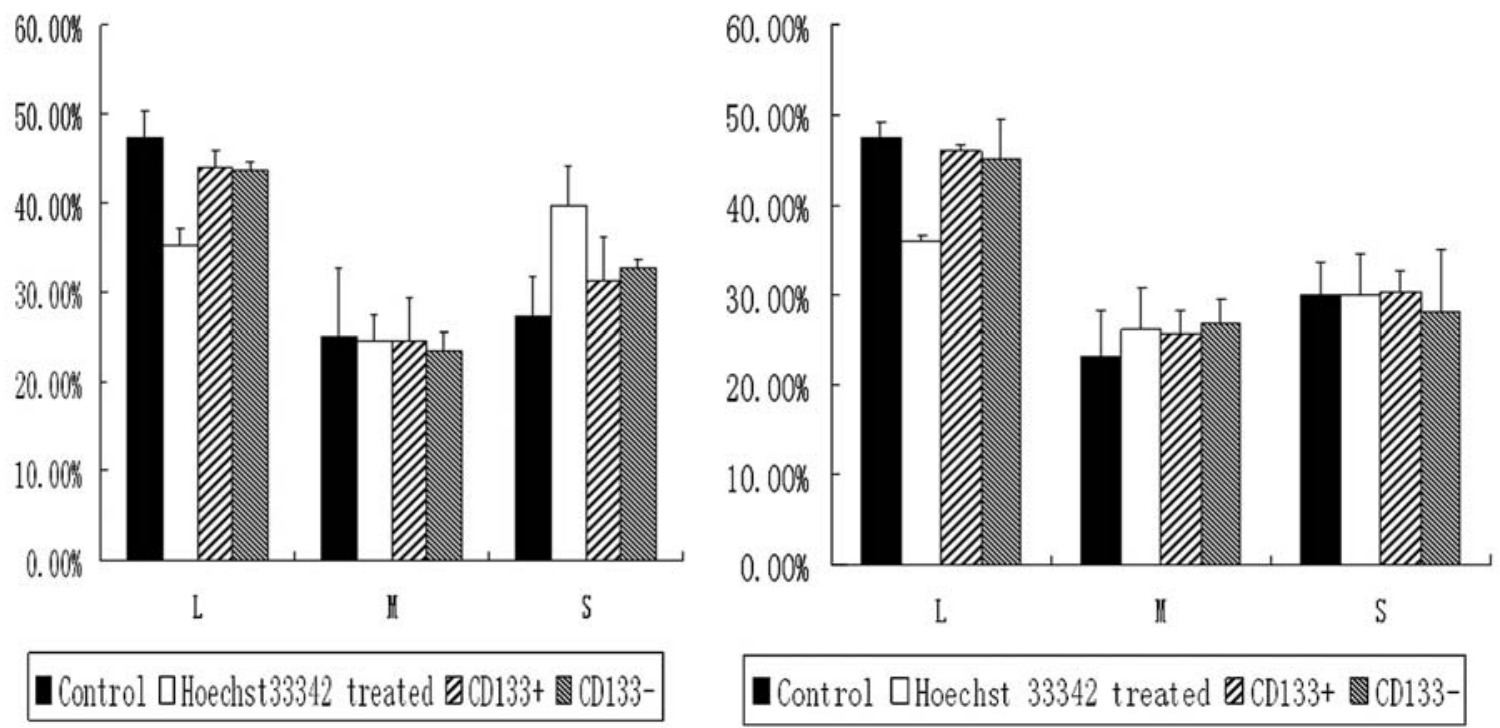

Figure 5. Comparison of the clone formation rates of different cells. The formation rates of the large clone of Hoechst 33342 -treated A549 and H446 cells were significantly lower than that of control. The $\mathrm{CD}_{133^{-}}$cells has the same formation rate of large cones as that of CD133 cells, which was just a little lower than that of the control.
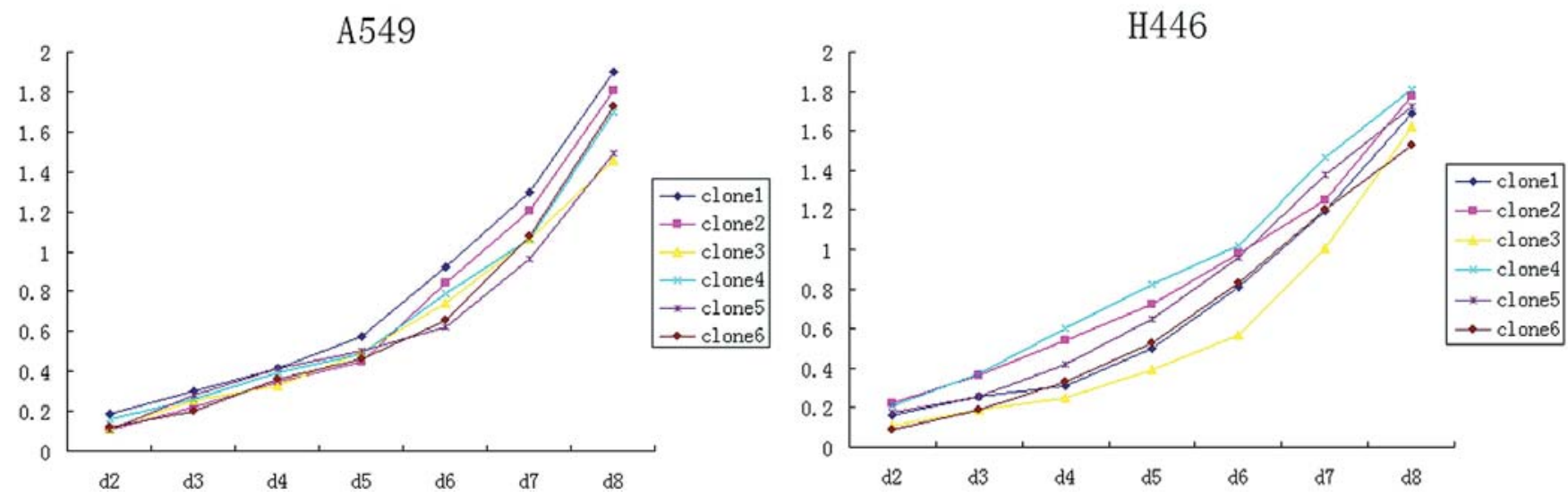

Figure 6. The growth curves of A549 and H446 cells.

99\% of Hoechst 33342-free H446 cells formed clones. The formation rate of large clones was significantly lower than that of the control $(\mathrm{P}<0.05)$. The formation rate of small clones was also significantly lower than that of the control $(\mathrm{P}<0.05)$ (Fig. 5).

Distribution of cell cycle phases. The percentages of A549 cells in G1, G2 and S phase were 43.39, 13.65 and 42.96\%, respectively. The percentages of $\mathrm{H} 446$ cells in G1, G2 and S phase were 62.75, 13.29 and 23.96\%, respectively (Fig. 4B).

Growth curve. The time needed for A549 cells to expand from one single cell to $10^{6}$ varied from 19 to 26 days. The time needed for H446 cells to expand from one single cell to $10^{6}$ varied from 17 to 25 days. The growth curves showed that different clones of A549 and H446 proliferated at the same rate when growing from one cell to $1 \times 10^{6}$ cells (Fig. 6).

\section{Discussion}

It was reported that only the cancer stem cells retain unlimited regeneration and tumorigenic ability, and most cancer cells do not possess tumorigenic ability (23). In contrast to the stem cells, the non-stem cells are thought to possess very limited regeneration ability (3-5). The A549 and H446 lung cancer cell lines have been used widely for decades and cultured in vitro for thousands of passages, but their tumorigenicity remains quite stable. A549 and H446 cell lines are immortal and proliferate quite rapidly. Their doubling time is $<24 \mathrm{~h}$ and we rarely found great cell loss in the A549 and 
H446 cell lines unless they had been contaminated or treated. Therefore, the number of proliferating cells is much larger than that of dormant cells. From this aspect, most cells of A549 and $\mathrm{H} 446$ possess regeneration capacity.

It was reported that only CD133+ cells contain stem cells in the lung cancer tissue (9). However, our result showed that both purified CD133+ and CD133- A549 and H446 cells could generate tumorigenic clones and there were no significant differences in the formation rates of large clones between $\mathrm{CD} 133^{+}$cells and CD133- cells. Flow cytometry showed that the fraction of $\mathrm{CD} 133^{+}$cells was much lower than that of clonogenic and tumorigenic cells. Similarly, the notion that non-SP cells are not stem cells is questionable (10). Incubation with $5 \mu \mathrm{g} / \mathrm{ml}$ Hoechst 33342 for 90 min severely affected their viability, clonogenicity and proliferation. Moreover, the flow cytometric procedure may further affect the stained cells. Therefore, Hoechst 33342 dye treatment in the efflux assay might be sufficient to cause the differences in clonogenicity and tumorigenicity between SP cells and non-SP cells.

Without any classification based on CD133 or ABCG2, single-cell cloning culture of unseparated A549 and H446 cells using routine procedures of end-point dilution showed that $>45 \%$ of A549 and H446 cells could generate large selfrenewable clones. Even after 10 passages, they still formed the three kinds of clones and the cells from large clones gave rise to a xenograft tumor in nude mice.

Cancer stem cell defines the tumor-forming ability of an undifferentiated cancer cell that might express normal stem cell markers without necessarily exhibiting other behaviors attributable to normal stem cells. It was proposed that a single cell that is capable of giving rise to a tumor of its own origin in vivo is termed a cancer stem cell $(13,24)$. According to this criterion, $>45 \%$ of viable $\mathrm{H} 446$ and A549 cells are cancer stem cells.

The cell cycle study showed that most cells were in G1 phase, which is contrary to the notion that the stem cells are in quiescence. However, the growth curves of different clones showed that once the number of cells reached $10^{6}$ from a single cell, they would proliferate at the same rate. These data suggested that most A549 and H446 cells may not be stem cells, but when they are alone they may acquire stem cell characteristics. Therefore, the cells that can form large clones should be called cancer initiating cell, not stem cells.

More than $45 \%$ of A549 and H446 single cells formed large clones, which underwent 10 passages and maintained their clonogenicity. The cells growing from one cell can form a xenograft tumor. It is still unclear what percentage of the A549 and H446 cells are cancer stem cells, but our results indicated that $>45 \%$ of A549 and H446 cells are cancer initiating cells.

In conclusion, we found that human lung cancer cell lines A549 and H446 contain $>45 \%$ of cancer initiating cells that can generate large clones, which were capable of giving rise to xenograft tumors in vivo. Both the CD133+ cells and the CD133- cells had the cancer initiating capacity. Moreover, we found that Hoechst 33342 decreased the clonogenicity and proliferation of a single A549 and H446 cell. Therefore, the process of Hoechst 33342 staining might inhibit the cancer initiating capacity of non-SP cells.

\section{References}

1. Singh SK, Clarke ID, Terasaki M, et al: Identification of a cancer stem cell in human brain tumors. Cancer Res 63: 5821-5828, 2003.

2. Singh SK, Hawkins C, Clarke D, et al: Identification of human brain tumor initiating cells. Nature 432: 396-401, 2004.

3. Kondo T, Setoguchi T and Taga T: Persistence of a small subpopulation of cancer stem-like cells in the C6 glioma cell line. Proc Natl Acad Sci USA 101: 781-786, 2004.

4. Al-Hajj M, Wicha MS, Benito-Hernandez A, Morrison SJ and Clarke MF: Prospective identification of tumorigenic breast cancer cells. Proc Natl Acad Sci USA 100: 3983-3988, 2003.

5. Collins AT, Berry PA, Hyde C, Stower MJ and Maitland NJ: Prospective identification of tumorigenic prostate cancer stem cells. Cancer Res 65: 10946-10951, 2005.

6. Suetsugu A, Nagaki M, Aoki H, Motohashi T, Kunisada T and Moriwaki H: Characterization of $\mathrm{CD}_{133^{+}}$hepatocellular carcinoma cells as cancer stem/progenitor cells. Biochem Biophys Res Commun 351: 820-824, 2006.

7. Yin S, Li J, Hu C, et al: CD133 positive hepatocellular carcinoma cells possess high capacity for tumorigenicity. Int J Cancer 120: 1444-1450, 2007.

8. Hermann PC, Huber SL, Herrler T, et al: Distinct populations of cancer stem cells determine tumor growth and metastatic activity in human pancreatic cancer. Cell Stem Cell 1: 313-323, 2007.

9. Eramo A, Lotti F, Sette G, et al: Identification and expansion of the tumorigenic lung cancer stem cell population. Cell Death Differ 15: 504-514, 2008.

10. Ho MM, Ng AV, Lams S and Hung JY: Side population in human lung cancer cell lines and tumors is enriched with stemlike cancer cells. Cancer Res 67: 4827-4833, 2007.

11. O'Brien CA, Pollett A, Gallinger S and Dick JE: A human colon cancer cell capable of initiating tumour growth in immunodeficient mice. Nature 445: 106-110, 2007.

12. Ricci-Vitiani L, Lombardi DG, Pilozzi E et al: Identification and expansion of human colon-cancer-initiating cells. Nature 445: 111-115, 2007.

13. Jodan CT: Cancer stem cell biology: from leukemia to solid tumors. Curr Opin Cell Biol 16: 708-712, 2004.

14. Liu G, Yuan X, Zeng Z, et al: Analyses of gene expression and chemoresistance of CD $133^{+}$cancer stem cells in glioblastoma. Mol Cancer 2;5:67, 2006.

15. Bao S, Wu Q, McLendon RE, et al: Glioma stem cells promote radioresistance by preferential activation of the DNA damage response. Nature 444: 756-760, 2006.

16. Beier D, Hau P, Proescholdt M, et al: CD133(+) and CD133(-) glioblastoma-derived cancer stem cells show differential growth characteristics and molecular profiles. Cancer Res 67: 4010-4015, 2007.

17. Shmelkov SV, Butler JM, Hooper AT, et al: CD133 expression is not restricted to stem cells, and both $\mathrm{CD}_{133^{+}}$and $\mathrm{CD} 133^{-}$ metastatic colon cancer cells initiate tumors. J Clin Invest 118 : 2111-2120, 2008.

18. Zheng X, Shen G, Yang X and Liu W: Most C6 cells are cancer stem cells: evidence from clonal and population analyses. Cancer Res 67: 3691-3697, 2007. Erratum in: Cancer Res 67: 10097, 2007.

19. Singh S, Dwarakanath BS and Mathew TL: DNA ligand Hoechst-33342 enhances UV induced cytotoxicity in human glioma cell lines. J Photochem Photobiol B 77: 45-54, 2004.

20. Singh S, Dwarakanath BS and Mathew TL: Role of topoisomerases in cytotoxicity induced by DNA ligand Hoechst 33342 and UV-C in a glioma cell line. Indian J Exp Biol 43: 313-323, 2005.

21. Atlasi Y, Mowla SJ, Ziaee SA and Bahrami AR: OCT-4, an embryonic stem cell marker, is highly expressed in bladder cancer. Int J Cancer 120: 1598-1602, 2007.

22. Hochedlinger K, Yamada Y, Beard C and Jaenisch R: Ectopic expression of Oct-4 blocks progenitor-cell differentiation and causes dysplasia in epithelial tissues. Cell 121: 465-477, 2005.

23. Setoguchi T, Taga T and Kondo T: Cancer stem cells persist in many cancer cell lines. Cell Cycle 3: 414-415, 2004.

24. Al-Hajj M, Becker MW, Wicha M, Weissman I and Clarke MF: Therapeutic implications of cancer stem cells. Curr Opin Genet Dev 14: 43-47, 2004. 